\title{
Politik der Leiblichkeit. Von Maurice Merleau-Ponty zu Iris Marion Young und Judith Butler
}

Steffen Herrmann, FernUniversität in Hagen

(final draft, erschienen in: Thomas Bedorf, Tobias N. Klaas (Hg.), Leib - Körper - Politik. Untersuchungen zur Leiblichkeit des Politischen, Weilerswist: Velbrück 2015, S. 61-82)

Eine der grundsätzlichen Thesen von Maurice Merleau-Ponty lautet, dass unser Zur-WeltSein nicht ausgehend von der Instanz des Bewusstseins, sondern ausgehend von der Instanz der Leibes zu verstehen ist. Auch wenn Merleau-Ponty aus dieser These selbst kaum politische Konsequenzen gezogen hat, sind seine Überlegungen doch im Feld der politischen Philosophie von einer ganzen Reihe von Theoretikerinnen und Theoretikern aufgenommen worden, von denen ich im Folgenden zwei aufgreifen möchte: Iris Marion Young und Judith Butler. Beide, so werden ich zeigen, knüpfen in ihren Überlegungen nicht nur direkt an Merleau-Pontys Überlegungen am, um zu zeigen inwiefern der Leib sowohl Objekt als auch Subjekt des politischen Handelns ist, zugleich auch entwickeln beide dessen Überlegungen in entscheidenden Hinsichten weiter: Während uns Young vor Augen führt, dass die Persistenz von politischen Machtverhältnissen ausgehend von der Eigensinnigkeit des Leibes verstanden werden kann, zeigt uns Judith Butler ausgehend von der Idee der Geschichtlichkeit der Leibes, dass dieser als ein Ort von transformativen Möglichkeiten verstanden werden kann. Ausgehend von diesen Anknüpfungen an Merleau-Ponty durch Young und Butler werde ich abschließend argumentieren, dass ,Weltlichkeit', ,Eigensinnigkeit' und ,Geschichtlichkeit' drei wesentliche Momente einer Politik der Leiblichkeit sind.

Maurice Merleau-Ponty gehört neben Jean-Paul Sartre und Simone de Beauvoir zu einer der wichtigsten philosophischen Figuren der französischen Philosophie der 1940er und 1950er Jahre. Obwohl ihn mit beiden über längere Zeit eine persönliche Freundschaft verbindet, die unter anderem zum gemeinsamen Engagement in der Gruppe „Socialisme et Liberte“ führt, gilt Merleau-Ponty als blassester Vertreter in diesem Dreigestirn. Der Grund hierfür ist wohl vor allem, dass Sartre und Beauvoir ihren theoretischen Überlegungen einen unmittelbaren politischen Gehalt verleihen, wohingegen Merleau-Ponty eher zurückhaltend ist - seine 1945 veröffentlichte Phänomenologie der Wahrnehmung hat nicht den politischen Verve und den kämpferischen Ton eines Sartre und einer Beauvoir. Es ist nicht zuletzt diesem Umstand geschuldet, dass Merleau-Ponty lange Zeit nicht als ein politischer Denker wahrgenommen wurde. Zu technisch schien sein Hauptwerk zu sein und zu wenig tagesaktuell sein Inhalt. In der großen Auseinandersetzung zwischen Ost und West, welcher die Zeitgenossen MerleauPontys beschäftigte, waren es eher die großen politischen Themen, welche auf breite Resonanz stießen - eine Theorie der Leiblichkeit schien zu dieser Auseinandersetzung zu wenig beitragen zu können, als dass sie große Beachtung gefunden hätte.

Dieser Eindruck hat sich im Laufe der Jahrzehnte freilich verändert: Mittlerweile sind Überlegungen zur Leiblichkeit in vielen politischen Theorien zu einem wichtigen Bestandteil geworden. ${ }^{1}$ Diese veränderte Einschätzung der Bedeutung von Merleau-Pontys Werk kann nicht zuletzt an den Auseinandersetzungen, die Iris Marion Young und Judith Butler zu Beginn der 1980er Jahre mit dessen Denken geführt haben, deutlich gemacht werden. ${ }^{2}$ Beide

1 Vgl. Emmanuel Alloa u.a. (Hg.), Leiblichkeit. Geschichte und Aktualität eines Konzepts, Tübingen: Mohr Siebeck UTB 2012, 23-36.

2 Die einschlägigen Aufsätze von Young und Butler finden sich in dem Band Jeffner Allen und Iris Marion Young, The Thinking Muse: Feminism and Modern French Philosophy, Bloomington: Indiana University Press 1989. 
argumentieren dafür, dass sich mithilfe der Theorie der Leiblichkeit untergründige politische Machtverhältnisse transparent machen und für Veränderungen öffnen lassen. Im Besonderen zeigt sich dies für beide am Geschlechterverhältnis: Vor dem Hintergrund von MerleauPontys Überlegungen lässt sich dieses nicht nur in seiner Historizität und Gewordenheit verstehen, sondern zugleich auch in seiner Persistenz und Beharrlichkeit. Anders gesagt: Young und Butler sind der Überzeugung, dass eine Theorie der Leiblichkeit sowohl für eine Theorie der Ungleichheit als auch für eine Theorie der Emanzipation wichtige Ansatzpunkte liefert.

Um diesen Gedanken deutlich zu machen, will ich zunächst noch einmal zu den Überlegungen von Merleau-Ponty zurückkehren. Im Zuge der Rekonstruktion der Grundlagen seiner Theorie der Leiblichkeit sollen zunächst deren politische Potenziale freigelegt werden (i). Im Anschluss daran werde ich mich dann Youngs Rezeption von Merleau-Ponty zuwenden und zeigen, wie sie die Theorie der Leiblichkeit für eine politische Analyse der gesellschaftlichen Situation der Frau fruchtbar macht (ii). Im dritten Schritt werde ich mich schließlich den Überlegungen von Butler zuwenden, um $\mathrm{zu}$ zeigen, inwiefern ihre Infragestellung der natürlichen Zweigeschlechtlichkeit eine neue Auffassung der Geschlechtlichkeit als Stil der Existenz ermöglicht (iii). Der Durchgang durch die Überlegungen von Merleau-Ponty, Young und Butler wird es schließlich erlauben, unter den Stichworten Weltlichkeit, Geschichtlichkeit und Eigensinnigkeit drei Grundzüge einer Politik der Leiblichkeit zu formulieren (iv).

\section{Die Entdeckung der Leiblichkeit: Maurice Merleau-Ponty}

Der Ansatz, gegen den Merleau-Ponty seine Theorie der Leiblichkeit in Stellung bringt, ist das von ihm sogenannte, objektive Denken'. ${ }^{3}$ Gemeint ist damit nichts anderes als die cartesianische Tradition mit ihrem Dualismus von Geist und Körper. Während für Descartes der Geist weder in die Sphäre des Körpers hineinreichen noch andersherum als etwas Körperliches verstanden werden kann, möchte Merleau-Ponty im Ausgang vom Begriff des Leibes eine Zwischensphäre freilegen. Die menschliche Existenz, so will er zeigen, ist in erster Linie ein leibliches Zur-Welt-sein und jede Theorie, die das übersieht, vernachlässigt ein grundlegendes Charakteristikum der menschlichen Realität. Was aber ist unter der leiblichen Existenzweise des Menschen zu verstehen? Die Antwort auf diese Frage möchte ich im Folgenden in drei Schritten rekonstruieren: Zunächst werde ich zeigen, wie uns Merleau-Ponty im Ausgang von einer Analyse des Phantomgliedes deutlich macht, dass die menschliche Existenz auf der Grundlage der Leiblichkeit des Subjekts verstanden werden muss. Im Anschluss daran soll sich zeigen, dass das leibliche Handeln die primäre Vollzugsform unseres alltäglichen Zur-Welt-seins bildet, um dann im letzten Schritt auf die Frage zu sprechen zu kommen, wie sich der Leib im Zuge einer Reihe von Praktiken der Verleiblichung konstituiert.

Das ,objektive Denken' findet sich in der Mitte des 20. Jahrhunderts aus der Perspektive Merleau-Pontys in zwei theoretischen Strömungen wieder: dem Physiologismus auf der einen und dem Psychologismus auf der anderen Seite. Beide stellen insofern Abkömmlinge des Cartesianismus dar, als sie jeweils eine der beiden Seiten der Geist-Körper-Trennung zum Ausgangspunkt ihrer Forschung machen: Während der Psychologismus unsere körperliche Existenzweise vom Primat des Geistes her versteht, versteht der Physiologismus unsere geistigen Zustände von den Körperempfindungen her. Dass beide Positionen unzureichend sind, um die menschliche Realität zu verstehen, zeigt Merleau-Ponty am Phänomen des

\footnotetext{
3 Merleau-Ponty, Maurice (1945), Phänomenologie der Wahrnehmung, hrsg. u. übers. v. Rudolf Boehm, Berlin: Wilhelm Fink 1974, 91. Ein instruktive Rekonstruktion von Merleau-Pontys Denken findet sich bei Bernhard Waldenfels, Das leibliche Selbst. Vorlesungen zur Phänomenologie des Leibes, Frankfurt/M.: Suhrkamp 2000.
} 
Phantomgliedes. ${ }^{4}$ Dieses zeigt sich derart, dass nach der operativen Entfernung eines Körpergliedes der Eindruck persistiert, das entsprechende Glied sei noch vorhanden und spürbar - ein Eindruck, der so lebhaft sein kann, dass beispielsweise eine beinamputierte Patientin auf ihrem Phantombein aufzutreten versucht und dadurch stürzt. Physiologismus und Psychologismus versuchen das Phänomen des Phantomgliedes von jeweils unterschiedlichen Seiten her zu verstehen: Der Physiologismus geht davon aus, dass das Phantomglied das Resultat einer Irritation der Nervenenden an der amputierten Stelle ist, durch welche unser Gehirn mit falschen Daten versorgt wird und daher davon ausgeht, dass das Glied noch vorhanden ist. Im Gegensatz dazu geht der Psychologismus davon aus, dass die betroffene Person, auch wenn sie um den Verlust ihres Gliedes weiß, diese Einsicht doch rational verweigert. Im einen Fall wird das Phantomglied also als Resultat einer Wahrnehmungstäuschung, im anderen als Resultat einer Willensschwäche gedeutet.

Merleau-Ponty hinterfragt die Überzeugungskraft beider Erklärungen, indem er die jeweils eine Seite mit den Argumenten der jeweils anderen Seite konfrontiert: So kann der Physiologismus nicht erklären, warum das Phantomglied von psychischen Determinanten abhängig ist. Er ist nicht in der Lage anzugeben, warum dieses mit zunehmender Verarbeitung des Verlustes durch die Betroffenen verschwindet. ${ }^{5}$ Denn wenn es sich beim Phantomglied nur um eine Wahrnehmungstäuschung handelte, dann dürften psychische Prozesse keine Rolle spielen. Der Psychologismus wiederum kann nicht erklären, wie es sein kann, dass das Phantomglied nach der Beseitigung der Nervenleitungen zum Gehirn verschwindet. ${ }^{6}$ Würde es sich beim Phantomglied nur um eine verweigerte Anerkennung des Verlustes handeln, dürfte ein physiologischer Eingriff keine Folgen haben. Eine einseitige physiologische oder psychologische Theorie scheint dem Phänomen des Phantomgliedes also nicht gewachsen zu sein. Weder lässt sich dieses allein als Wahrnehmungstäuschung noch als Willensschwäche beschreiben, ohne dabei in Widersprüche zu geraten. Die Aufklärung des Phantomgliedes benötigt daher einen alternativen Verständnisrahmen.

Merleau-Ponty versucht einen solchen Verständnisrahmen mithilfe einer ,existentiellen Analyse' im Anschluss an Sartre zu liefern. ${ }^{7}$ Die Grundidee einer solchen Analyse besteht darin, dass das Subjekt der Welt nicht gegenübersteht, sondern immer schon in diese verstrickt ist. Wir nehmen niemals einen neutralen Beobachterstandpunkt ein, von dem aus wir dann auf die Welt zugehen; vielmehr sind wir immer schon auf ein bestimmtes Vorhaben hin ausgerichtet. Merleau-Ponty spricht diesbezüglich vom „Zur-Welt-sein“. Dieses meint, dass sich uns die Welt je schon von einer bestimmten Seite her zeigt: So taucht die Eiche auf dem Feld für den pflügenden Bauern als ein Hindernis auf, während sie der flanierenden Spaziergängerin ein willkommenes schattiges Plätzchen zum Rasten bietet. Je nachdem also, in welches Projekt sich ein Subjekt gerade engagiert findet, zeigt sich ihm die Welt auf ganz bestimmte Weise. Folgt Merleau-Ponty in der Anlage seiner existenziellen Analyse bis hierhin noch grundsätzlich dem Denken Sartres, so setzt er sich von diesem in eben jenem Moment ab, in welchem er davon ausgeht, dass das engagierte Ich nicht allein auf der Ebene des Bewusstseins, sondern auch auf derjenigen des Organismus angesiedelt ist. Um diesen Gedanken plausibel zu machen, zieht er das Konzept des Leibes heran. Mit ihm will er deutlich machen, dass der Organismus nicht einfach ein Reiz-Reaktions-Apparat ist, der sensorische Daten empfängt und weiterleitet, sondern dass er vielmehr selbst bereits eine sinnhaft organisierte Einheit bildet. In ein Projekt engagiert zu sein bedeutet nämlich nicht nur, dass das Bewusstsein auf eine Tätigkeit fokussiert ist. Zugleich auch ist der ganze Organismus auf diese Tätigkeit ausgerichtet: Er befindet sich in einer gewissen tonischen Spannung, seine Gliedmaßen nehmen eine bestimmte Haltung ein und seine Motorik ist in

Merleau-Ponty, Phänomenologie der Wahrnehmung, $100 \mathrm{ff}$.

Ebd.

Ebd., 101.

Ebd., 159. 
Bereitschaft, spezifische Aktionen auszuführen. Der Organismus, so drückt es Merleau-Ponty aus, ist auf die Welt als einem „Feld von Möglichkeiten“ ausgerichtet. ${ }^{8}$ Und diese Intentionalität ist für Merleau-Ponty nicht mehr sinnvoll als Resultat von bewussten Prozessen $\mathrm{zu}$ verstehen - wir bringen unseren Körper nur selten bewusst in eine ganz bestimmte Lage -, im Gegenteil zeugt sie von einer sinnhaften und präreflexiven Organisation des Organismus im Rahmen des Zur-Welt-seins. Es ist eben diese Dimension unserer Existenz, die Merleau-Ponty mit dem Begriff des Leibes zu fassen versucht. ${ }^{9}$ Und in diesem Sinne ist der Leib nicht mit dem Körper zu verwechseln: Während letzterer die materielle Faktizität des Organismus meint, meint ersterer dessen sinnhafte Gesamtorganisation.

Kommt man mit dem entwickelten begrifflichen Hintergrund nun auf das Phantomglied zurück, dann lässt sich dieses auf neue Weise verstehen. War in der cartesianischen Perspektive nur die Wahl zwischen einem Physiologismus, der das Phantomglied als Wahrnehmungstäuschung versteht, und einem Psychologismus, der es als Willensschwäche deutet, geblieben, so eröffnet sich nun mit einer existenziellen Analyse eine dritte Sichtweise: Das Phantomglied ist Ausdruck der Tatsache, dass der Leib für die Möglichkeiten des verlorenen Gliedes in der Welt noch offen bleibt. Was den Verlust verweigert, ist weder das sensible, reizbare Ich, noch das bewusste, wollende Ich, sondern der Leib in seiner Gerichtetheit auf die Welt als einem Feld von Möglichkeiten. Merleau-Ponty spricht hier davon, dass die dem „aktuellen Leib“ nicht mehr möglichen Gesten noch im „habituellen Leib“ aufbewahrt sind. ${ }^{10}$ Der vergebliche Griff nach der Tasse oder das Abstützen mit dem Bein zeugen davon, dass die neue Gesamtorganisation des Organismus sich noch nicht habitualisiert hat. Ausgehend von dieser Analyse vermag Merleau-Ponty nun die Erklärungsschwierigkeiten $\mathrm{zu}$ lösen, an denen Physiologismus und Psychologismus gescheitert sind. Er kann nämlich sowohl erklären, warum das Phantomglied mit zunehmender Dauer verschwindet - nämlich aufgrund der Ausbildung eines neuen leiblichen Habitus -, als auch, wieso das Phantomglied durch die Entfernung der Nervenleiter zum Verschwinden gebracht werden kann - insofern der leibliche Habitus nicht in der Luft hängt, sondern von den vom Stumpf ausgehenden Erregungen zehrt. ${ }^{11}$ Die Sphäre des Leibes erweist sich nun insofern als eine Zwischensphäre, als der Leib weder einfach einen auf kausale ReizReaktionsmechanismen reduzierten Körper meint, noch auf ein sich selbst transparentes Bewusstsein zurückgeführt werden kann. Vielmehr handelt es sich um eine Sphäre eigener Sinnhaftigkeit, die sowohl an der Faktizität des Körpers als auch an der Transzendenz des Bewusstseins partizipiert.

Nachdem Merleau-Ponty den Begriff des Leibes in Auseinandersetzung mit dem Phantomglied im ersten Schritt eingeführt hat, geht es ihm nun im zweiten Schritt darum zu zeigen, dass die leibliche Existenzweise die fundamentalste Struktur der menschlichen Realität ist. Deutlich macht er diesen zweiten Punkt am sogenannten „Fall Schneider“. ${ }^{12}$ Bei Schneider handelt es sich um einen kleinhirngeschädigten Kriegsveteran, der seit seiner Kopfverletzung unter einer Reihe von motorischen Einschränkungen leidet. Schneiders koordinative Schwierigkeiten lassen sich dabei entlang von zwei Klassen von Bewegungen gruppieren: den sogenannten konkreten und abstrakten Bewegungen. Mit konkreten

\footnotetext{
$8 \quad$ Ebd., 194.

9 Merleau-Ponty spricht auch vom Körperschema - ein Begriff, der mehr oder weniger synonym zum Begriff des Leibes verwendet werden kann, jedoch terminologisch irreführend ist. Dass Merleau-Ponty nicht von einem leiblichen Schema spricht, ist der Tatsache geschuldet, dass es sich beim Begriff des Körperschemas um einen von Paul Schilder etablierten terminus technicus handelt. Vgl. hierzu auch ausführlich. Stefan Kristensen „Maurice Merleau-Ponty - Körperschema und leibliche Subjektivität“, in: Emmanuel Alloa u.a. (Hg.), Leiblichkeit. Geschichte und Aktualität eines Konzepts, Tübingen: Mohr Siebeck UTB 2012, 23-36.

$10 \quad$ Ebd.; Merleau-Ponty, Phänomenologie der Wahrnehmung, 107.

11 Ebd., 111

12 Ebd., $128 \mathrm{ff}$
} 
Bewegungen sind die in das alltägliche Geschehen eingebetteten Handlungen des Organismus gemeint: Ein Taschentuch aus der Hosentasche ziehen, um die Nase zu schnäuzen, ein Streichholz entfachen, um eine Lampe anzuzünden oder an einer juckenden Körperstelle kratzen, um sich Erleichterung zu verschaffen. Alle diese lebensweltlich eingebetteten Tätigkeiten stellen für Schneider keine Herausforderung dar. Anders dagegen verhält es sich mit abstrakten Bewegungen: Auf Kommando einen militärischen Gruß ausführen, auf die eigene Nasenspitze zu zeigen oder Pantomime zu spielen. Alle diese Tätigkeiten, die nicht in eine konkrete Situation eingebettet sind, sondern in Bezug auf eine fiktive Situation im Modus des Als-ob vollzogen werden müssen, stellen für Schneider eine große Herausforderung dar. Im Gegensatz zum sogenannten Normalen, der sich spielerisch und problemlos in solche Situationen versetzen und seinen Organismus entsprechend bewegen kann, ist Schneider zu den entsprechenden Bewegungen nur schwerlich in der Lage.

Merleau-Ponty deutet den Fall Schneider nun so, dass dieser abstrakte Bewegung nicht mehr leiblich, sondern nur noch intellektuell zu bewältigen vermag. Das bedeutet, dass er in fiktiven Situationen ganz und gar auf den Status eines cartesischen Subjekts zurückgeworfen ist. Die Aufforderung einen militärischen Gruß zu vollziehen, hat für ihn keine unmittelbare leibliche Bedeutung mehr, vielmehr muss er diese Aufforderung erst mit dem Verstand verarbeiten: Er muss die Lage seiner Glieder mithilfe seines Blicks feststellen, um dann im Anschluss seine Glieder in die vorgegebene Geste zu lenken. Wie schwierig ein solcher instrumenteller Gebrauch des Körpers zur Durchführung einer Geste ist, zeigt sich daran, dass Schneider mehrere langwierige Versuche unternehmen muss, bis es ihm gelingt, den geforderten Gruß durchzuführen. Anders verhält es sich in aktuellen Situationen: Juckt es Schneider am Oberarm, so kann er sich problemlos an der genannten Stelle kratzen. Er muss nicht erst, wie im Fall von abstrakten Bewegungen, die zugehörige Bewegung mühsam intellektuell entwerfen. Vielmehr bilden die juckende Hautstelle und die kratzende Hand hier eine Einheit, in der beide unmittelbar aufeinander bezogen sind. Was Schneider in fiktiven Situationen also fehlt, ist jene Fähigkeit, die Merleau-Ponty an den Begriff der Leiblichkeit gebunden hatte: die Orientierung des Organismus auf ein Feld von Möglichkeiten hin.

Das Auseinandertreten von abstrakten und konkreten Bewegungen im Fall Schneider macht deutlich, dass sich beim ,Normalen' ein großer Teil des alltäglichen Handelns nicht vorsätzlich und bewusst, sondern unwillkürlich und präreflexiv vollzieht. Merleau-Ponty spricht davon, dass Leib und Welt im leiblichen Handeln zu einem „intentionalen Bogen“ zusammengeschlossen sind. ${ }^{13}$ Einerseits ist der Leib am Entwurf der Welt als einem Feld von Möglichkeiten beteiligt (wie im Fall des Phantomgliedes) und andererseits vermag er auf Anforderungen der Welt unmittelbar zu antworten (wie im Fall der konkreten Bewegungen). Der Fall Schneider hat für Merleau-Ponty also die Aufgabe, die sozialphilosophischen Konsequenzen der existenzialistischen Sichtweise auf den Organismus deutlich zu machen. Während das cartesianische Subjekt seinen Organismus in dem Sinn hat, dass sein Körper vom Bewusstsein besessen und gesteuert wird und dieser allein als Instrument des Handelns zu verstehen ist, ist das existenziale Subjekt sein Organismus in dem Sinne, dass der Leib als eigenständige präreflexive Handlungsinstanz verstanden wird, durch die ein Großteil von alltäglichen Handlungssituationen bewältigt wird.

Nachdem Merleau-Ponty mithilfe des Phantomgliedes die Instanz des Leibes als eine Zwischensphäre des Zur-Welt-seins eingeführt und dann am Fall Schneider die Bedeutung dieser Zwischensphäre für unser alltägliches Dasein herausgekehrt hat, gilt es für ihn noch die Frage zu beantworten, wie sich der habituelle Leib durch Praktiken der Verleiblichung konstituiert. Es ist eben dieser letzte Zug seiner Überlegungen, der seinen Gedanken ihr kritisches Potential verleiht: Weit davon entfernt den Leib als natürliches Gattungsmerkmal zu verstehen, deutet Merleau-Ponty ihn als eine „geschichtliche Idee“. ${ }^{14}$ Die leiblichen

$13 \quad$ Ebd., 159.

14 Ebd., 203 
Schemata, die wir im Zuge unseres Zur-Welt-seins in Anschlag bringen, deutet er also nicht als Ausdruck einer natürlichen Fähigkeit, sondern als Effekte von Praktiken der Verleiblichung. Solche Praktiken, so Merleau-Ponty, lassen sich weder ausgehend vom Intellektualismus noch vom Behaviorismus begreifen. Ein leibliches Schema zu erwerben bedeutet nämlich weder sich ein intellektuelles Wissen anzueignen, das dann als Regel in Anschlag gebracht wird, noch bedeutet es eine einfache Abrichtung auf bestimmte Reize. Gegen den Intellektualismus spricht, dass leibliches Wissen präreflexiv ist und im Zweifelsfall gar nicht explizit gemacht werden kann - Merleau-Ponty führt diesbezüglich das Schreiben auf einer Schreibmaschine an, das beherrscht wird, ohne explizit angeben zu können, wo sich welche Buchstaben auf der Klaviatur befinden. ${ }^{15}$ Gegen den Behaviorismus spricht wiederum, dass ein einmal erlerntes leibliches Schema auf vielfältige Situationen angewendet werden kann und nicht auf die Lernsituation beschränkt bleibt - so kann beispielsweise eine Orgelspielerin ihr Können auf ganz unterschiedlichen Instrumenten zur Geltung bringen. Wenn also weder Intellektualismus noch Behaviorismus hinreichend erklären können, wie der habituelle Leib zustande kommt, wie müssen wir uns seinen Erwerb dann vorstellen?

Merleau-Pontys Antwort auf diese Frage lautet, dass wir ein leibliches Schema durch Gewöhnung erlernen. Wir lernen, wie etwas zu tun ist, indem wir etwas wieder und wieder tun. Dabei kann explizites Wissen vorläufig eine durchaus tragende Rolle spielen. Wer etwa lernen will, die Orgel zu spielen, der muss sich mit den Tasten und Registern vertraut machen, ihre Bedeutung lernen und zu Beginn genau darauf achten, wie und wo er genau seine Glieder in Stellung bringt. Im Verlauf des Repetierens tritt dieses explizite Moment jedoch immer weiter in den Hintergrund: Das Wissen lagert sich nach und nach in das leibliche Schema ein. Ist die Organistin nun gezwungen auf einer neuen Orgel zu spielen, so ist kein Vermessen der Abstände zwischen den Registern und Tasten ihrerseits notwendig - vielmehr spielt sie sich an der neuen Orgel ein, um Leib und Instrument aufeinander abzustimmen und sich die neuen Relationen einzuverleiben. Im Begriff der Gewöhnung steckt daher nicht umsonst der Begriff des Wohnens. Er verweist darauf, dass die Lernende nach und nach am Instrument heimisch wird und ohne Hemmungen und Stockungen zu spielen vermag. Gewöhnung meint also nicht die Einverleibung von exakten Bewegungsabläufen, sondern vielmehr bestimmte Strukturen und Relationen leiblich kennen und beherrschen zu lernen. Der Leib, so Merleau-Ponty, erwirbt durch Gewöhnung das Vermögen, „Situationen gewisser Gestalt in Lösungen eines gewissen Typs zu entsprechen, wobei die Situationen von einem Fall zum anderen sehr verschiedene sein können“. ${ }^{16}$

Die leibliche Eingewöhnung im Umgang mit bestimmten Dingen hat noch eine weitere Folge: Sind wir mit einem bestimmten Gegenstand vertraut geworden, vermag dieser eine besondere, gleichsam ,magische“ Anziehungskraft auszuüben. So wird diejenige, die mit der Orgel vertraut ist und ein solches Instrument in neuer Umgebung antrifft, automatisch von diesem angesprochen werden. Das Instrument fordert den Leib gleichsam dazu auf, sich seiner zu bedienen. Die Eingewöhnung bestimmter leiblicher Praktiken strukturiert das Zur-Welt-sein des Subjekts also in ganz bestimmter Weise. Sie versetzt es, so können wir sagen, in eine Situation. Und es ist eben dieses leibliche Situiertsein, von dem der kritische Impuls von Merleau-Pontys Philosophie ausgeht. Deutlich wird das ganz zum Schluss der Phänomenologie der Wahrnehmung, wenn sich Merleau-Ponty kritisch mit Sartres Philosophie der Freiheit auseinandersetzt. Das präreflexive Zur-Welt-sein, so argumentiert er hier, bildet die Grenze, an welcher die Freiheit des Cogito beständig zu scheitern droht. Freilich, so gesteht Merleau-Ponty zu, vermögen wir prinzipiell stets mit unserer Vergangenheit zu brechen und uns auf neue Weise zu wählen. Stets jedoch muss die Freiheit

$15 \quad$ Ebd., 173.

16 Ebd., 172 
dabei mit der Macht der Gewohnheit ringen: „,[U]nsere Situation ist, solange wir leben, eine offene, was zugleich besagt, daß sie privilegierte Weisen ihrer Auflösung fordert" ${ }^{\text {“ }}{ }^{17}$

Ausgehend von diesem Überblick über Merleau-Pontys Theorie der Leiblichkeit wird deutlich, dass die kritische Stoßrichtung seiner Überlegungen in zwei Richtungen geht: Einerseits geht es ihm darum, den Leib als eine geschichtliche Idee und nicht als eine natürliche Tatsache zu denken. Diese entnaturalisierende Stoßrichtung von Merleau-Pontys Überlegungen wird Judith Butler in der Folge aufgreifen und radikalisieren. Andererseits geht es Merleau-Ponty darum, die gesellschaftliche Situiertheit des Leibes zu denken. Unsere leibliche Existenz ist mit einer ganz bestimmten Gravitationskraft verbunden, die unserer Freiheit Grenzen setzt. Eben diese zweite Stoßrichtung wird in der Folge von Iris Marion Young aufgenommen und weiter ausgearbeitet werden.

\section{Die Zurichtung des Leibes: Iris Marion Young}

Einen entscheidenden Meilenstein in der kritischen Aneignung von Merleau-Pontys Theorie der Leiblichkeit bildet Iris Marion Youngs Aufsatz mit dem provokanten Titel „Werfen wie ein Mädchen“" aus dem Jahr 1980. Young versucht hier die gesellschaftskritischen Potenziale einer Theorie der Leiblichkeit für die feministische Theoriebildung fruchtbar zu machen. Diese, so Young, habe dem Leib bisher zu wenig Aufmerksamkeit geschenkt, was nicht zuletzt daran liege, dass Simone de Beauvoir in ihrer einschlägigen Studie Das andere Geschlecht den Körper als Ort der Unfreiheit der Frau gebrandmarkt habe. Insofern Beauvoir den weiblichen Körper als Last beschreibt, durch welchen die Frau an der Transzendenz und damit an der Verwirklichung ihrer Individualität gehindert wird, droht sie die weibliche Anatomie zur Ursache der Erfahrung der Frau zu machen. Young dagegen glaubt, dass die Lasterfahrung des weiblichen Körpers keine natürlichen Ursachen hat, sondern als Effekt der kulturellen Zurichtung des weiblichen Körpers $\mathrm{zu}$ verstehen ist. Merleau-Pontys Ausführungen zur Theorie der Leiblichkeit stellen für sie dabei ein ausgezeichnetes theoretisches Mittel dar, diese spezifisch weibliche Erfahrung als Effekt der „Situation der Frauen" deutlich zu machen. ${ }^{18}$

Young beginnt ihren Aufsatz mit der Diskussion einer Studie, die zeigt, dass es große Unterschiede im Wurfverhalten von Jungen und Mädchen gibt: Während der Junge eine Wurfbewegung unter dem Einsatz seines ganzen Körpers vollzieht, indem er den Arm zur Seite nimmt, das Bein nach hinten stellt und seinen Rumpf zur Seite dreht, bleibt das Mädchen wie angewurzelt auf der Stelle stehen. Es hebt lediglich ihren Arm in die Horizontale und bringt den Unterarm in eine gebeugte Position. Dieses Verhalten hat zur Folge, dass dort, wo der Junge einen Wurf mit Kraft und Präzision auszuführen vermag, er sich beim Mädchen ohne Kraft und Zielgebung vollzieht. Das hier beschriebene Wurfverhalten der beiden Geschlechter gilt Young nicht als Einzelfall, sondern als Exempel dafür, dass sich bei Männern und Frauen im Allgemeinen zwei ganz unterschiedliche Formen des motorischen Körperverhaltens ausmachen lassen. ${ }^{19}$ Dabei zeigt sich in allen alltäglichen und zweckgerichteten Aktionen, dass Frauen im Vergleich zu Männern zu einem gehemmten Körperverhalten neigen: So sind die Schritte des Mannes im Verhältnis zu seiner Körpergröße insgesamt größer als diejenigen der Frau, so sitzt er dort, wo die Frau die Beine nahe aneinander presst, gerne breitbeinig oder ebenso steht er gerne gerade und stämmig, während die Frau eher eine geschwungene Haltung einnimmt. Kurz: Männer und Frauen zeigen zwei ganz unterschiedliche Stile des Körperverhaltens; während dasjenige des Mannes frei und unumschränkt wirkt, scheint das der Frau gebunden und gehemmt.

\footnotetext{
$17 \quad$ Ebd., 502.

18 Iris Marion Young, „Werfen wie ein Mädchen“, in DZPhil 41, 1993/4, 707-725, 722. Vgl. dazu ebenso dies., „Throwing Like a Girl: Twenty Years Later“, in: Donn Welton (Hg.), Body and Flesh. A Philosophical Reader, Oxford: Blackwell 1998, 286-290.

19 Young, „Werfen wie ein Mädchen“, 712
} 
Um die genannten weiblichen Körpererfahrung in ein theoretisches Vokabular zu übersetzen, wendet sich Young in einem zweiten Schritt den Überlegungen von Merleau-Ponty zu. Im Anschluss an dessen leibtheoretische Untersuchungen beschreibt sie die praktischen Körpererfahrungen der Frau als ,gehemmte Intentionalität“ und als „mehrdeutige Transzendenz“ ${ }^{20}$ Mit dem Begriff der gehemmten Intentionalität will sie darauf hinweisen, dass der weibliche Leib sich zwar auf ein Ziel in der Welt ausrichtet, in eins mit dieser Ausrichtung jedoch zugleich dieses Ziel in unerreichbare Ferne rückt. Im Ausgang von Merleau-Pontys Analyse des Phantomgliedes hatten wir bereits gesehen, dass der Leib die Welt als ein Feld von Möglichkeiten erschließt. Mit dem Aufscheinen von solchen Möglichkeiten ist freilich auch immer das Aufscheinen von Widerständen verbunden - erst dort nämlich, wo sich der Leib auf eine Aufgabe hin organisiert, vermögen ihm dieser Aufgabe entgegenstehende Widrigkeiten zu erscheinen. Während in der männlichen Existenz das zweite Element nun weitgehend im Hintergrund zu bleiben scheint, tritt es in der weiblichen Existenz in den Vordergrund. Stellt der Leib eigentlich eine Domäne dar, durch welche sich die Welt als ein Feld von Möglichkeiten eröffnet, so ist diese Öffnung für die Frau gleichzeitig mit einer Schließung verbunden: An die Stelle des leiblichen „Ich kann“, welches die Welt als Aktionsraum konstituiert, tritt ein „Ich kann nicht“, welches die bestehenden Handlungsoptionen verschließt.

Verbunden mit dieser gehemmten Intentionalität tritt jenes Phänomen auf, dass Young als mehrdeutige Transzendenz beschreibt: Gemeint ist damit, dass der weibliche Körper sich nicht als Ganzer zur Welt hin öffnet, sondern immer nur partiell auf diese gerichtet ist. Nur ein Teil des Körpers richtet sich auf die Aufgabe des Zur-Welt-seins, während der Rest gehemmt bleibt. Eben dieses Auseinandertreten von leiblichem Zur-Welt-sein und körperlichem Verhaftetsein beschreibt Young dann schließlich als „nicht kontinuierliche Einheit“ der weiblichen Existenz. ${ }^{21}$ Der weibliche Organismus, so will sie damit sagen, scheint von einer Spaltung durchzogen zu sein: Während sich ein Teil von ihm auf die Welt hin orientiert, bleibt ein anderer Teil auf sich selbst gerichtet. Anders gesagt: Der weibliche Organismus lebt in einem Spannungsverhältnis von „Leib sein“ und „Körper haben“. Das erinnert in gewissem Sinne an den oben besprochenen Fall Schneider: Konnte sich dieser im Fall von konkreten Bewegungen problemlos leiblich in der Welt orientieren, so war er im Fall von abstrakten Bewegungen ganz auf seinen Körper zurückgeworfen, den er wie ein Instrument handhaben musste, um die geforderten Bewegungen auszuführen. Während bei Schneider der Bruch zwischen „Leib sein“ und „Körper haben“ jedoch an zwei ganz unterschiedliche Situationen gebunden war, tritt der Bruch in der weiblichen Existenz in ein und derselben Situation auf: In derselben Bewegungen findet sich die Frau zugleich als Leib und als Körper wieder - und eben daraus resultieren jene gehemmten motorischen Verhaltensweisen, wie wir sie im Fall des Werfens kennengelernt haben.

Freilich, so argumentiert Young in einem dritten Schritt, ist der Riss, der durch die Existenz der Frau verläuft und ihre Erfahrung prägt, nicht als Ausdruck einer spezifisch weiblichen Natur zu verstehen. Vielmehr muss die leibliche Gehemmtheit als Ausdruck einer Reihe von vergeschlechtlichten Praktiken verstanden werden, durch welche die Heranwachsende ihre weibliche Geschlechterrolle einübt. ${ }^{22}$ Im losen Anschluss an die Untersuchungen von Young lassen sich dabei fünf unterschiedliche Register von Praktiken unterscheiden. ${ }^{23}$ Als physiotechnische Praktiken lassen sich all jene Praktiken beschreiben, die unter die Haut gehen, um die Zell-, Gewebe- oder Muskelstruktur des Körpers zu verändern. Im Mittelpunkt stehen hier Fitness- und Wellness-Programme, durch welche die Frau einen möglichst

\footnotetext{
$20 \quad$ Ebd., $715 \mathrm{f}$.

Ebd., $717 f$.

Ebd., 723.

Vgl. dazu Steffen Herrmann, „Ein Körper werden. Praktiken des Geschlechts“, in: A.G.Gender-Killer (Hg.), Das gute Leben, Linke Perspektiven auf einen besseren Alltag, Münster: Unrast 2007, 13-32.
} 
jugendlichen und grazilen Körper ausbilden soll. Territorialisierende Praktiken sind jene Praktiken, welche auf der Oberfläche des Körpers ansetzen. Mithilfe von kosmetischen Produkten soll hier die Ausdruckskraft des Körpers gesteigert werden. Fragmentierende Praktiken wiederum legen sich wie eine zweite Haut über den Körper. Kleidung und Schmuck dienen dazu, bestimmte Partien des Körpers wie etwa Oberkörper, Taille und Beine hervortreten $\mathrm{zu}$ lassen. Transgressive Praktiken betreffen die Beziehung des weiblichen Körpers zum Raum. Hierher gehören die von Young selbst genannten Beispiele des Sitzens, Stehens und Gehens. Als soziale Praktiken lassen sich schließlich alle Praktiken bezeichnen, welche den Umgang des Mädchens mit den Dingen der Welt und anderen Menschen betreffen. Zentral sind hier all jene Geschlechternormen, durch welche die Frau als Objekt des (männlichen) Blicks und als Subjekt der (mütterlichen) Sorge konstituiert wird.

Wir haben es insgesamt mit einer Reihe ganz unterschiedlicher Praktiken zu tun, durch welche die Frau auf ihren Körper einwirkt und welche zur Folge haben, dass sie eine ganz bestimmte leibliche Existenzweise ausbildet. Deutlich wird damit, dass Weiblichkeit keine natürliche Eigenschaft ist, welche irgendwie aus der Anatomie des Körpers entspringt, sondern vielmehr eine Existenzweise bezeichnet, die durch eine Reihe von Praktiken eingeübt und hergestellt werden muss. Weiblichkeit, so lässt sich festhalten, ist der Effekt einer Reihe von Praktiken, durch welche ein bestimmter leiblicher Habitus inkorporiert wird. Freilich lässt sich dabei mit Young betonen, dass sich ein solcher leiblicher Habitus nicht bei jeder Frau zeigen muss, dass er aber dort, wo er nicht zu finden ist, auf negative Weise bestimmend ist als etwas, dem eine Frau entkommen musste. ${ }^{24}$ Das gleiche lässt sich auf den Einwand entgegnen, dass sich ein weiblicher leiblicher Habitus auch bei Männern finden lassen wird auch hier wirkt er negativ bestimmend als etwas, dem sich der Mann aktiv gegen Widerstände zuwenden muss. Letztlich weisen solche Beispiele geschlechtlicher Devianz nur darauf hin, dass die Übereinstimmung eines bestimmten leiblichen Habitus mit einer bestimmten Anatomie eine arbiträre Angelegenheit ist. Von Natur aus ist keine Frau weiblich und kein Mann männlich, vielmehr sind Weiblichkeit und Männlichkeit die Effekte einer gesellschaftlichen Regulation von geschlechtlichen Praktiken - es ist eben dieser Punkt, den Judith Butler in der Folge weiter ausarbeiten wird.

Kommen wir zuvor jedoch nochmals auf die Auswirkungen dieser gesellschaftlichen Regulation von Geschlechterpraktiken zurück. Für Young besteht ein wesentlicher Effekt dieser Praktiken darin, dass sich die Frau im Laufe ihrer Sozialisation als Objekt für den männlichen Blick konstituieren muss. Sie wird zu einem Körper, der von anderen gesehen, taxiert und bewertet wird. Indem sie derart beständig mit ihrem eigenen Gesehenwerden konfrontiert ist, nimmt sie nach und nach eine Außenperspektive auf sich ein. ${ }^{25}$ Eben das hat aber zur Folge, dass sie nicht mehr unmittelbar leiblich in der Welt aufzugehen vermag. Denn in dem Moment, in dem sie ihren Körper zum Gegenstand des Blicks macht, unterbricht sie ihren unmittelbaren leiblichen Weltbezug. Denn während der Leib das Medium einer unmittelbaren und präreflexiven Kommunikation mit der Welt ist, ist der Körper das Instrument und der Gegenstand einer bewussten Handlung. ,Leib sein' und ,Körper haben' sind daher zwei gegenläufige existenzielle Einstellungen. Die Frau ist für Young in der modernen Gesellschaft nun dazu gezwungen, diese gegenläufigen Existenzweisen zu leben: Sie muss ihren Körper haben, während sie Leib ist. Die darin angelegte Widersprüchlichkeit drückt sich in der weiblichen Existenz als fortwährende leibliche Gehemmtheit aus. Jene Lasterfahrung, die Simone de Beauvoir in Das andere Geschlecht mit dem weiblichen Körper verbunden hat, so lässt sich damit im Anschluss an Young argumentieren, ist nicht das Resultat der Anatomie der Frau, sondern das Resultat der Art und Weise, wie der weibliche Körper in der modernen Gesellschaft zugerichtet wird.

\footnotetext{
24 Young, „Werfen wie ein Mädchen“, 722.

25 Ebd., 724
} 
Young vermag mit ihrer feministischen Aneignung von Merleau-Pontys Theorie der Leiblichkeit jedoch nicht nur, die von Beauvoir beschriebenen Lasterfahrungen als gesellschaftlichen Effekt der Konstitution von Weiblichkeit einzuholen, sie vermag darüber hinaus auch noch, eine weitere Frage schlüssig zu beantworten, die Beauvoir in Das andere Geschlecht stellt. Nämlich die Frage danach, warum die Frau nicht gegen ihre Unterdrückung aufbegehrt, sondern sich mit ihrer Rolle abfindet. „Woher“, so fragt Beauvoir, „kommt diese Unterwerfung bei der Frau?“. ${ }^{26}$ Youngs Überlegungen erlauben es uns, eine Antwort auf diese Frage zu geben: Die Frau vermag aus ihrer gesellschaftlichen Rolle deshalb nicht einfach auszubrechen, weil es sich dabei nicht bloß um eine dramatische Rolle handelt, von der jederzeit Abstand genommen werden kann - etwa so wie die Theaterspielerin jederzeit ihre Rolle ablegen und zu einem agierenden Subjekt werden kann. Vielmehr muss die gesellschaftliche Rolle der Frau im Ausgang von ihrer leiblichen Existenzweise begriffen werden - ihr Rollenskript steht ihr nicht gegenständlich vor Augen, sondern hat sich vielmehr in ihr Fleisch eingeschrieben. Anders gesagt: Es gibt ein Verhaftetsein an die gesellschaftlichen Geschlechternormen: Und zwar nicht, weil es eine Identifikation oder ein Einverständnis mit diesen Normen geben würden, sondern weil diese Normen durch eine Reihe von vergeschlechtlichten Praktiken leiblich wirksam sind. Die Frau, so können wir im Anschluss an Young festhalten, hat es also deshalb so schwer, sich aus ihrer gesellschaftlichen Situation zu befreien, weil das ganze Gewicht des leiblichen Habitus einer solchen Befreiung entgegensteht.

\section{Die Normativität des Leibes: Judith Butler}

Neben Iris Marion Young hat sich auch Judith Butler in den 1980er Jahren in zwei Aufsätzen mit den Überlegungen von Merleau-Ponty auseinandergesetzt. ${ }^{27}$ Dessen Theorie der Leiblichkeit hält sie für derart überzeugend, dass sie zu dieser Zeit noch für einen „phänomenologischen Feminismus“ eintritt. ${ }^{28}$ Dabei ist ihre Rezeption von Merleau-Ponty durchaus ambivalent: Während seine Theorie für sie einerseits eine radikale gesellschaftliche Sprengkraft birgt, insofern sie es erlaubt, den Leib jenseits von naturalistischen Vorstellungen als ein kulturelles Erzeugnis zu denken, fällt seine Anwendung dieser Theorie auf den geschlechtlichen Leib weit hinter dieses kritische Potenzial zurück, da sie dazu tendiert, bestehende Geschlechternormen zu naturalisieren. Es ist der letzte Punkt, der Butler dazu veranlasst hat, dem Projekt eines phänomenologischen Feminismus nicht auf Dauer treu zu bleiben, sondern sich vielmehr einem diskurstheoretischen Feminismus zuzuwenden, da letzterer aus ihrer Perspektive die konzeptuellen theoretischen Mittel bereitstellt, eine Reihe von gesellschaftlichen Normen in den Blick zu nehmen, die dem phänomenologischen Ansatz Merleau-Pontys verschlossen bleiben mussten. Ausgehend von diesem Blickwechsel, so werde ich im Folgenden zeigen, wird Butler Merleau-Pontys Idee der Geschichtlichkeit des Leibes in ein neues Licht rücken.

Butlers Rezeption von Merleau-Pontys Theorie der Leiblichkeit in ihrem Aufsatz „Geschlechtsideologie und phänomenologische Beschreibung“ ist zunächst eine kritische.

26 Simone de Beauvoir, Das andere Geschlecht. Sitte und Sexus der Frau (1949), Hamburg: Rowohlt $2000,14$.

27 Judith Butler, „Geschlechtsideologie und phänomenologische Beschreibung. eine feministische Kritik an Merleau-Pontys Phänomenologie der Wahrnehmung“, in: Silvia Stoller und Helmuth Vetter (Hg.), Phänomenologie und Geschlechterdifferenz, Wien: WUV-Universitätsverlag, 166-186; dies., „Performative Akte und Geschlechterkonstitution. Phänomenologie und feministische Theorie“, in: Uwe Wirth (Hg.) Performanz. Zwischen Sprachphilosophie und Kulturwissenschaften, Frankfurt/ Main: Suhrkamp, S. 301-320. Eine ausführliche Auseinandersetzung mit dem Verhältnis von Butler und Merleau-Ponty findet sich in der aufschlussreichen Studie von Silvia Stoller, Existenz - Differenz - Konstruktion,. Phänomenologie der Geschlechtlichkeit bei Beauvoir, Irigaray und Butler, München: Wilhelm Fink 2010. Vgl. ebenso: Thomas Bedorf, „Leibliche Praxis. Zum Körperbegriff der Praxistheorien“, in: Thomas Alkemeyer, Volker Schürmann u. Jörg Volbers (Hg.), Praxis [denken]. Konzepte und Kritik, Wiesbaden: Springer VS 2004.

28 Butler, „Geschlechterideologie“, 182. 
Einerseits behauptet dieser nämlich, es gehe ihm darum, den Leib als eine geschichtliche Idee zu denken, andererseits fällt er dort, wo es diese Idee praktisch zu denken gilt, weit hinter sie zurück. Exemplarisch zeigt sich das für Butler an der Analyse des Falls Schneider. MerleauPonty geht davon aus, dass bei Schneider eine Störung des geschlechtlichen leiblichen Schemas vorliegt und führt dafür folgende drei Beobachtungen ins Feld: Erstens rufen obszöne Bilder oder der Anblick eines Körpers bei ihm keinerlei Begierden hervor, zweitens bringt der Orgasmus seiner Partnerin seine Begierde zum Verschwinden und drittens findet Schneider Frauen nicht aufgrund ihres Körpers, sondern aufgrund ihres Charakters anziehend. ${ }^{29}$ Unschwer, so Butler, sind die Vorurteile zu entziffern, die in die jeweiligen Beispiele einfließen: So bleibt im ersten Fall völlig unhinterfragt, welche kulturellen Bedingungen gegeben sein müssen, damit sexuelle Erregung beim Anblick von obszönen Bildern als normal gilt. Im zweiten Fall wird Schneiders Rücksichtnahme auf seine Partnerin als sexuelle Unfähigkeit gedeutet und so davon ausgegangen, dass das männliche Begehren völlig unabhängig vom Zustand der Partnerin besteht. Im dritten Fall schließlich scheint Merleau-Ponty davon auszugehen, dass der weibliche Körper ein Wesen besitzt, dass den männlichen Körper unmittelbar anzieht und dessen Begehren herausfordert. Merleau-Ponty geht in seinen Beispielen also nicht nur durchweg von einer natürlichen Heterosexualität aus, zugleich auch scheint die aktive und bemächtigende Rolle des Mannes und die passive Rolle der Frau für ihn selbstverständlich zu sein. Was nun, so fragt Butler, wenn Schneider homosexuell gewesen wäre? Und was, wenn er Feminist gewesen wäre und seine Haltungen nicht als Störungen des geschlechtlichen Körperschemas, sondern vielmehr als emanzipative Rekonfigurationen desselben zu denken sind? ${ }^{30}$ Eine solche Möglichkeit vermag MerleauPonty nicht zu denken, da er in jener geschlechtlichen Matrix gefangen bleibt, für deren Auflösung seine Theorie doch eigentlich die entscheidenden begrifflichen Werkzeuge liefert. In der Praxis erweist sich Merleau-Ponty daher nicht dazu in der Lage, den Leib als jene geschichtliche Idee zu denken, die den Ausgangspunkt seiner Überlegungen zu den Techniken der Verleiblichung bildet. Dieser praktische Mangel verweist für Butler auf ein grundsätzliches Defizit von Merleau-Pontys phänomenologischer Herangehensweise. Die Theorie der Leiblichkeit, so lautet Butlers Vorwurf, bleibe in einem expressiven Modell des Selbst gefangen, insofern sie davon ausgeht, dass sich ein geschlechtliches Selbst in seinen leibliches Praktiken zum Ausdruck bringe. Sie geht davon aus, dass wir es mit einem Subjekt $\mathrm{zu}$ tun haben, das geschichtlich mit einer Reihe von Ausdrucksmöglichkeiten seiner Geschlechtlichkeit konfrontiert sei und zwischen diesen zu wählen habe. Damit aber, so Butler, vermag die Phänomenologie die Geschichtlichkeit des Subjekts nur in einem losen Sinne zu denken. ${ }^{31}$ Demgegenüber argumentiert Butler, dass die Geschichtlichkeit des Subjekts in einem starken Sinn gedacht werden muss: Das Selbst geht den geschlechtlichen Praktiken nicht vorher, sondern wird durch diese zuallererst hervorgebracht.

Obwohl Butler sowohl praktische als auch theoretische Kritik an Merleau-Pontys Überlegungen übt, bleibt sie der Theorie der Leiblichkeit gleichwohl wohlgesonnen. Jedoch gilt es für sie, die Idee der Geschichtlichkeit des Leibes auf radikalere Art und Weise zu denken. Für Butler bedeutet das, den phänomenologischen Theorierahmen hinter sich zu lassen und sich einer Theorie der Performativität zuzuwenden. Im Zentrum der Theorie des Performativen, wie Butler sie zum ersten Mal in ihrem Aufsatz „Performative Akte und Geschlechterkonstitution“ vorstellt, steht daher das Vorhaben zu zeigen, „daß das, was als Geschlechteridentität bezeichnet wird, eine performative Leistung ist, die durch gesellschaftliche Sanktionen und Tabus erzwungen wird“. ${ }^{32}$ Geschlechtsidentität versteht

\footnotetext{
29 Merleau-Ponty, Phänomenologie der Wahrnehmung, $186 \mathrm{f}$.

30 Butler, „Geschlechtsideologie“, 175f. und 185.

31 Eine ausführliche Auseinandersetzung und Widerlegung von Butlers Einwänden finden sich in Sylvia Stoller, Existenz- Differenz - Konstruktion, $391 \mathrm{ff}$.

32 Butler, „Performative Akte“, 302.
} 
Butler hier, der amerikanischen Tradition gemäß, im Ausgang von den Begriffen von sex und gender als Übereinstimmung zwischen biologischem Geschlecht und sozialer Geschlechtszugehörigkeit. Butler argumentiert nun, dass jene Vollzüge, durch welche wir eine Geschlechtszugehörigkeit in Szene setzen, nicht als Ausdruck des biologischen Geschlechts zu verstehen sind. Die Konstitution der Geschlechtszugehörigkeit ist nicht expressiv, sondern performativ zu verstehen. Deutlich wird das am Beispiel der Travestie: Sie führt uns vor Augen, dass unsere Geschlechtszugehörigkeit der Effekt einer Reihe von rituellen Dramatisierungen ist. Deutlich wird so, dass die Zugehörigkeit zu einem Geschlecht nur in dem Maße anerkannt wird, wie sie performiert wird. Es ist eben diese produktive und erzeugende Kraft menschlichen Handelns, welche Butler mit dem Begriff des Performativen in den Mittelpunkt rückt. Gleichzeitig betont sie jedoch auch, dass die Dramatisierung der Geschlechtszugehörigkeit nicht auf einer individuellen Wahl beruht, sondern durch gesellschaftliche Normen reguliert ist: in erster Linie durch die Norm der Übereinstimmung zwischen sex und gender. Während die provokative Zurschaustellung der Inkongruenz von biologischem Geschlecht und sozialem Geschlecht auf der Bühne jedoch aufgrund künstlerischer Konventionen akzeptiert wird und jederzeit mit der Bemerkung abgewehrt werden kann, dass es sich ja ,nur' um ein Schauspiel handle, sind inkongruente Vollzüge der Geschlechtsidentität im Alltag gefährlich: Hier wird auf die Nichtübereinstimmung von sex und gender zumeist mit drastischen Sanktionen reagiert. ${ }^{33}$ Das zeigt sich nicht zuletzt daran, dass in der westlichen Kulturgeschichte der weibliche Mann und die männliche Frau zumeist Spottgestalten bilden.

Folgen wir Butlers Überlegungen bis zu diesem Punkt, so zeigt sich, dass sie mit den Analysen von Iris Marion Young übereinstimmen. Hier wie dort geht es darum, Geschlechtlichkeit als Effekt einer Reihe von gesellschaftlichen Praktiken zu verstehen. Phänomenologische und performative Analyse scheinen daher zum selben Ergebnis zu führen. Mehr noch: Die phänomenologische Analyse scheint den großen Vorteil zu haben, ein reichhaltigeres Vokabular zur Beschreibung der leiblichen Effekte von geschlechtlichen Praktiken und darüber hinaus eine Beschreibung unseres Zur-Welt-seins anbieten zu können, in denen leibliche Vollzüge einen zentralen Stellenwert einnehmen. Warum also die phänomenologische Analyse überhaupt verlassen? Worin liegt der Mehrwert, den sich Butler durch den Wechsel von einer phänomenologischen zu einer performativen Perspektive verspricht? Auf diese Frage ist zweierlei zu antworten: Zum einen erlaubt es die performative Analyseebene sich von der individuellen Erfahrungsebene zu lösen und die gesellschaftliche Konstitution der geschlechtlichen Normen von Weiblichkeit und Männlichkeit in den Blick zu nehmen. Es erlaubt Butler sich den überindividuellen Strukturen zuzuwenden, durch welche Geschlechternormen eingesetzt und aufrechterhalten werden. Zum anderen sieht Butler in der performativen Analyse die Möglichkeit, das feministische Projekt noch einmal auf eine ganz neue Stufe zu heben. Feministinnen, so Butler, sollten nämlich nicht nur an der Reproduktion der Geschlechtszugehörigkeit kritisch gegenüberstehen, sondern auch der Reproduktion der scheinbar klar abgegrenzten Kategorien von Mann und Frau. ${ }^{34}$ Anders gesagt: Es gilt für die feministische Theorie nicht nur zu zeigen, dass Weiblichkeit und Männlichkeit leibliche Effekte von kulturellen Praktiken sind, sondern zugleich auch die Kategorien von Frau und Mann selbst infrage zu stellen.

Es ist eben der letztgenannte Punkt, der Butlers Überlegungen zu weitreichender Prominenz verholfen und $\mathrm{zu}$ teilweise heftigen Widersprüchen geführt hat. Indem Butler nicht nur gender, sondern auch sex als Effekt von kulturellen Praktiken verstehen wolle, so wurde entgegnet, leugne sie die Faktizität des biologischen Geschlechts, die doch jede und jeder von

33 Vgl. Steffen Herrmann, »Bühne und Alltag. Über zwei Existenzweisen des Drag«, in: Pia Thilmann, Tania Witte u. Ben Rewald (Hg.), Drag Kings. Mit Bartkleber gegen das Patriarchat, Querverlag: Berlin 2007, $115-132$

$34 \quad$ Butler, „Performative Akte“, 308. 
uns morgens im Spiegel betrachten könne. Nun stellt Butler aber spätestens in ihrem Werk Körper von Gewicht deutlich klar, dass es ihr nicht um die Infragestellung von anatomischen Unterschieden zwischen Körpern geht. Vielmehr möchte sie mit der Infragestellung der Kategorie des biologischen Geschlechts darauf hinweisen, dass auch die Natur des körperlichen Geschlechts eine Geschichte besitze. ${ }^{35}$ Das adäquate Mittel für eine solche Analyse findet Butler in der Diskurstheorie Michel Foucaults. Dessen (und andere) Arbeiten zur Geschichte der Sexualität zeigen, dass Sexualität keine natürliche Angelegenheit ist, sondern sich die Vorstellung zweier natürlicher biologischer Geschlechter historisch erst langsam herausgebildet hat. ${ }^{36}$ Anders als Foucault und andere widmet sich Butler in ihren Überlegungen jedoch weniger einer historisch gesättigten Diskursanalyse der Zweigeschlechtlichkeit als vielmehr den diskursiven Bedingungen, unter denen so etwas wie das biologische Geschlecht überhaupt Gegentand $\mathrm{zu}$ werden vermag. Körperliche Unterschiede, so Butler, sind uns nie auf natürliche Art und Weise gegeben, sondern sie kommen immer nur innerhalb von kulturellen Deutungssystemen zur Erscheinung. Es verhält sich daher nicht so, dass wir zuerst zwei phänotypisch verschiedene Körperformen betrachten und diesen dann eine kulturelle Bedeutung beilegen. Wir haben es nicht zuerst mit einer natürlichen und anschließend mit einer kulturellen Deutung des Körpers zu tun. Vielmehr erweist sich der scheinbar neutrale naturwissenschaftliche Blick als immer schon mit kulturellen Vorannahmen durchsetzt. Wenn sex aber immer nur im Rahmen von gender adressiert werden kann, dann kann daraus gefolgert werden, dass das System der Zweigeschlechtlichkeit keine vorgängige natürliche Tatsache darstellt, sondern vielmehr durch eine Reihe von regulierenden Normen und Diskurse zuallererst hervorgebracht und aufrecht erhalten werden muss.

Kommen wir nun auf die Frage zurück, was Butlers Überlegungen zu einer Politik der Leiblichkeit beizutragen haben. Butler geht über die Analyse von Merleau-Ponty und Young in der Frage hinaus, wie die Geschichtlichkeit der Leibes und des Körpers zu denken ist. Das gelingt ihr in erster Linie durch einen Ebenenwechsel. Nicht mehr die Konstitution der individuellen Erfahrung, sondern die gesellschaftliche Konstitution von Intelligibilität tritt in ihren Überlegungen in den Mittelpunkt. Anders gesagt: Nicht die Existenzweise des Subjekts, sondern seine gesellschaftliche Existenzbedingungen gilt es zu untersuchen. Leiblichkeit und Körperlichkeit, so zeigt uns Butler, sind durch ein Geflecht von gesellschaftlichen Normen hervorgebracht, das für die Übereinstimmung zwischen biologischem Geschlecht (Körper) und Geschlechtszugehörigkeit (Leib) entlang der Achse der Zweigeschlechtlichkeit sorgt. Das Herausarbeiten der gesellschaftlichen Konstitution des Körpers hat für eine Theorie der Leiblichkeit nun insofern entscheidende Folgen, als die Einübung eines bestimmten leiblichen Habitus durch eine Reihe von Praktiken zustande kommt, die sich am System der Zweigeschlechtlichkeit orientieren. Wird nun eben jenes System als Effekt einer Reihe von gesellschaftlichen Normen entlarvt, dann verliert es seinen strukturierenden Einfluss auf jene Praktiken, durch welche ein geschlechtlicher Leib hervorgebracht wird. Dadurch eröffnet sich ein Möglichkeitsraum für neue Geschlechtsidentitäten, die sich statt an der jeweiligen anatomischen Ausstattung an der jeweiligen geschlechtlichen Performanz orientieren.

\section{Die Politik der Leiblichkeit}

Kommen wir nun noch einmal auf die Ausgangsfrage nach den gesellschaftskritischen Potenzialen von Merleau-Pontys Theorie der Leiblichkeit zurück, dann lassen sich drei Grundzüge einer Politik der Leiblichkeit hervorheben, die sich im Zuge der Rekonstruktion

\footnotetext{
35 Butler, Körper von Gewicht. Die diskursiven Grenzen des Geschlechts (1993), Frankfurt: Suhrkamp 1997.

36 Michel Foucault, Sexualität und Wahrheit: Erster Band: Der Wille zum Wissen (1976), Frankfurt/M.: Suhrkamp 1983. Einschlägig ist darüber hinaus die Studie von Thomas Laquer, Auf den Leib geschrieben. die Inszenierung der Geschlechter von der Antike bis Freud, Frankfurt/M. u. New York: Campus 1992.
} 
von Merleau-Ponty herauskristallisiert haben, und die in der Folge von Iris Marion Young und Judith Butler aufgenommen und weiter zugespitzt worden sind.

Merleau-Ponty hat uns zunächst die Weltlichkeit des Leibes vor Augen geführt. Der Einsatzpunkt seiner Überlegungen besteht darin, den Leib als eine dritte Instanz jenseits von Geist und Körper zu verstehen. Er zeigt, dass ein Großteil der Handlungen, die wir alltäglich in der Welt vollziehen, weder als Leistung des Bewusstseins noch als Reiz-ReaktionsMechanismen aufzufassen sind, sondern sich vielmehr auf der präreflexiven Ebene des Leibes vollziehen. Zweitens war es die Geschichtlichkeit des Leibes, die Merleau-Ponty ins Zentrum seiner Überlegungen gestellt hat. Der Leib, so hat er gezeigt, ist nicht als eine vorsoziale und natürliche Instanz zu verstehen, sondern als Resultat einer Reihe von Praktiken der Eingewöhnung. Den Leib sozialtheoretisch zu untersuchen, bedeutet daher, sich jenen gesellschaftlichen Arrangements und Institutionen zuzuwenden, die unseren Leib langfristig prägen und ihm so eine bestimmte Existenzweise einverleiben. Drittens hat Merleau-Ponty die Eigensinnigkeit des Leibes herausgestellt. Gemeint ist damit, dass der Leib zwar zu spontanen Handlungen in der Lage ist und kreativ auf immer neue Anforderungen zu reagieren vermag, dass er dabei aber bestimmten Mustern folgt. Dem durch Praktiken der Eingewöhnung hervorgebrachten leiblichen Habitus kommt eine Beharrlichkeit zu, an dem der freie Entwurf von Möglichkeiten beständig seine Grenze findet.

Weltlichkeit, Geschichtlichkeit und Eigensinnigkeit stellen also die drei kritischen Einsatzpunkte von Merleau-Pontys Theorie der Leiblichkeit dar. Nicht zufällig hat er sich daher im Ausgang von ihnen mit den marxistischen Ansätzen seiner Zeit auseinandergesetzt. Im Ausgang von der Weltlichkeit des Leibes hat er beispielsweise dafür argumentiert, dass das Klassenbewusstsein des Proletariats weder ausgehend von dessen intellektueller Reflexion noch von dessen objektiven Existenzbedingungen hinreichend beschrieben werden kann, sondern vielmehr als geteilte leibliche Existenzweise verstanden werden muss. ${ }^{37}$ Während Merleau-Ponty seine Theorie der Leiblichkeit damit in erster Linie in Bezug auf das Klassenverhältnis anzuwenden versucht hat, steht bei Iris Marion Young und Judith Butler das Geschlechterverhältnis im Vordergrund. Die Konsequenzen, die diesbezüglich aus der Theorie der Leiblichkeit für das politische Handeln zu ziehen sind, sind für beide aber nicht weniger grundlegend. Mit Merleau-Ponty sind sich beide zunächst darin einig, dass die Weltlichkeit der Welt im Ausgang von der leiblichen Existenz des Subjekts gedacht werden muss. Das Geschlechterverhältnis lässt sich weder auf die physische noch auf die psychische Ausstattung der Betroffenen zurückführen, vielmehr muss es als ein leibliches Verhältnis verstanden werden. Ausgehend von dieser Übereinstimmung entwickeln Young und Butler je einen Punkt von Merleau-Pontys kritischen Ansatzpunkten weiter.

Young, so haben wir gesehen, wendet sich im Ausgang von der Eigensinnigkeit des Leibes der Struktur von Ungleichheitsverhältnissen zu. Dabei vermag sie nicht nur zu zeigen, dass Frauen in der modernen Gesellschaft eine paradoxale Existenz führen müssen, indem sie immer wieder dazu genötigt werden, ihre leibliche Existenzweise zu verdinglichen und sich als Objekt des männlichen Blicks zu setzen. Des Weiteren zeigt uns Young auch, dass der leibliche Habitus den eigentlichen Ort geschlechtlicher Ungleichheitsverhältnisse bildet, insofern die herrschenden Geschlechternormen hier in inkorporierter Form wirksam sind. Die Persistenz der Geschlechternormen, so argumentiert sie, hat seine Ursache weniger darin, dass sie von den Betroffenen nicht angemessen durchschaut werden, als vielmehr darin, dass sie ihnen als leiblicher Habitus in Form einer zweiten Natur zu eigen ist. Die gesellschaftliche Situation der Frau kann für Young daher so lange nicht ausreichend erfasst werden, wie sie lediglich als Effekt von physischer Unterwerfung oder intellektueller Manipulation gefasst wird. Das Geschlechterverhältnis basiert daher weder auf Zwang noch auf falschem Bewusstsein, sondern auf der leiblichen Inkorporation von Geschlechternormen. 
Während Young im Ausgang von der Eigensinnigkeit des Leibes die Beschaffenheit von geschlechtlichen Ungleichheitsverhältnissein in den Blick nimmt, widmet sich Judith Butler im Ausgang von der Geschichtlichkeit des Leibes den emanzipativen Potenzialen der Theorie der Leiblichkeit. Butler radikalisiert dabei Merleau-Pontys Denken der Geschichtlichkeit des Leibes, indem sie die gesellschaftlichen Bedingungen der geschlechtlichen Intelligibilität zum Gegenstand ihrer Überlegungen macht. Derart gelingt es ihr, jene vorauslaufenden Bedingungen der Konstitution des Körpers in den Blick zu nehmen, welche die Grundlage der Verleiblichung bilden. Ist die Gültigkeit der binären körperlichen Ordnung aber erst einmal infrage gestellt, dann besteht die Geschichtlichkeit des Leibes nicht mehr einfach in einem Spielraum von historischen Interpretationsmöglichkeiten der Zweigeschlechtlichkeit, vielmehr werden ganz neue Weisen des geschlechtlichen Seins denkbar. Wenn sich das biologische Geschlecht selbst als eine kulturelle Tatsache entpuppt, dann gibt es keinen guten Grund mehr, die Reihe möglicher Geschlechtsidentitäten auf zwei zu begrenzen. Vielmehr lassen sich im Anschluss an eine Theorie der Leiblichkeit genau so viele mögliche Geschlechtsidentitäten denken, wie es leibliche Stile der Existenz gibt.

Spitzen wir abschließend zu: Was uns die Überlegungen von Young und Butler vor Augen führen ist, dass die Theorie der Leiblichkeit für die politische Praxis zwei grundsätzliche Anknüpfungspunkte bietet: Stimmen wir mit Merleau-Ponty darin überein, dass die menschliche Realität grundsätzlich vor dem Hintergrund der Weltlichkeit des Leibes verstanden werden muss, dann erlaubt es die Theorie der Leiblichkeit einerseits, Ungleichheitsverhältnisse als Verhältnisse der leiblichen Herrschaft auf neue Art und Weise zu denken und andererseits eröffnet sie uns die Möglichkeit, unsere Identität jenseits unserer anatomischen Ausstattung als einen leiblichen Stil der Existenz zu verstehen.

\section{Literatur:}

Allen, Jeffner, Young, Iris Marion (1989), The Thinking Muse: Feminism and Modern French Philosophy, Bloomington: Indiana University Press.

Alloa, Emmanuel u.a. (2012), Hg., Leiblichkeit. Geschichte und Aktualität eines Konzepts, Tübingen: Mohr Siebeck UTB 2012, 23-36.

Bedorf, Thomas (2014), „Leibliche Praxis. Zum Körperbegriff der Praxistheorien“, in: Thomas Alkemeyer, Volker Schürmann u. Jörg Volbers (Hg.), Praxis [denken]. Konzepte und Kritik, Wiesbaden: Springer VS.

Butler, Judith (1993), Körper von Gewicht. Die diskursiven Grenzen des Geschlechts, Frankfurt/M.: Suhrkamp.

Butler, Judith (1997), „Geschlechtsideologie und phänomenologische Beschreibung. Eine feministische Kritik an Merleau-Pontys Phänomenologie der Wahrnehmung“, in: Silvia Stoller u. HelmuthVetter (Hg.), Phänomenologie und Geschlechterdifferenz, Wien: WUVUniversitätsverlag, 166-186.

Butler, Judith (2002), „Performative Akte und Geschlechterkonstitution. Phänomenologie und feministische Theorie“, in: Uwe Wirth (Hg.) Performanz. Zwischen Sprachphilosophie und Kulturwissenschaften, Frankfurt/ Main: Suhrkamp, S. 301-320.

de Beauvoir, Simone (1949), Das andere Geschlecht. Sitte und Sexus der Frau, Hamburg 2000 .

Foucault, Michel (1976), Sexualität und Wahrheit: Erster Band: Der Wille zum Wissen, Frankfurt/M.: Suhrkamp 1983. 
Herrmann, Steffen (2007), „Bühne und Alltag. Über zwei Existenzweisen des Drag“, in: Pia Thilmann, Tania Witte u. Ben Rewald (Hg.), Drag Kings. Mit Bartkleber gegen das Patriarchat, Berlin: Querverlag, 115-132.

Herrmann, Steffen (2007), „Ein Körper werden. Praktiken des Geschlechts“, in: A.G.GenderKiller (Hg.), Das gute Leben, Linke Perspektiven auf einen besseren Alltag, Münster: Unrast, 13-32.

Kristensen, Stefan (2012), „Maurice Merleau-Ponty - Körperschema und leibliche Subjektivität", in: Emmanuel Alloa u. a. (Hg.), Leiblichkeit. Geschichte und Aktualität eines Konzepts, Tübingen: Mohr Siebeck UTB, 23-36.

Laquer, Thomas (1992), Auf den Leib geschrieben. die Inszenierung der Geschlechter von der Antike bis Freud, Frankfurt/M. u. New York: Campus.

Merleau-Ponty, Maurice (1945), Phänomenologie der Wahrnehmung, hrsg. u. übers. v. Rudolf Boehm, Berlin: Walter de Gryter 1974.

Stoller, Silvia (2010), Existenz - Differenz - Konstruktion. Phänomenologie der Geschlechtlichkeit bei Beauvoir, Irigaray und Butler, München: Wilhelm Fink 2010.

Waldenfels, Bernhard (2000), Das leibliche Selbst. Vorlesungen zur Phänomenologie des Leibes, Frankfurt/M.: Suhrkamp.

Young, Iris Marion (1993), „Werfen wie ein Mädchen“, in DZPhil 41, 4, 707-725.

Young, Iris Marion (1998), „Throwing Like a Girl: Twenty Years Later“, in: Donn Welton (Hg.), Body and Flesh. A Philosophical Reader, Oxford: Blackwell , 286-290. 